Chapman University

Chapman University Digital Commons

Psychology Faculty Articles and Research

Psychology

5-31-2017

\title{
Source Localization of Brain States Associated with Canonical Neuroimaging Postures
}

Michael Lifshitz

McGill University

Robert T. Thibault

McGill University

Raquel R. Roth

McGill University

Amir Raz

ChapmanUniversity, raz@chapman.edu

Follow this and additional works at: http://digitalcommons.chapman.edu/psychology_articles

Part of the Medical Neurobiology Commons, and the Neurosciences Commons

\section{Recommended Citation}

Lifshitz, M., Thibault, R. T., Roth, R. R., \& Raz, A. (2017). Source localization of brain states associated with canonical neuroimaging postures. Journal of Cognitive Neuroscience, 29(7), 1292-1301. https://doi.org/10.1162/jocn_a_01107

This Article is brought to you for free and open access by the Psychology at Chapman University Digital Commons. It has been accepted for inclusion in Psychology Faculty Articles and Research by an authorized administrator of Chapman University Digital Commons. For more information, please contactlaughtin@chapman.edu. 


\section{Source Localization of Brain States Associated with Canonical Neuroimaging Postures}

\section{Comments}

This article was originally published in Journal of Cognitive Neuroscience, volume 29, issue 7, in 2017. DOI: 10.1162/jocn_a_01107

\section{Copyright}

MIT Press 


\title{
Source Localization of Brain States Associated with Canonical Neuroimaging Postures
}

\author{
Michael Lifshitz ${ }^{1,2 *}$, Robert T. Thibault ${ }^{1,2 *}$, Raquel R. Roth $^{1}$, and Amir $\operatorname{Raz}^{1,2,3}$
}

\begin{abstract}
Cognitive neuroscientists rarely consider the influence that body position exerts on brain activity; yet, postural variation holds important implications for the acquisition and interpretation of neuroimaging data. Whereas participants in most behavioral and EEG experiments sit upright, many prominent brain imaging techniques (e.g., fMRI) require participants to lie supine. Here we demonstrate that physical comportment profoundly alters baseline brain activity as measured by magnetoencephalography (MEG) — an imaging modality that permits multipostural acquisition. We collected resting-state MEG data from 12 healthy participants in three postures (lying supine, reclining at $45^{\circ}$, and sitting upright). Source-modeling analysis revealed a broadly distributed influence of posture on resting brain function. Sitting upright versus lying supine was associated with greater highfrequency (i.e., beta and gamma) activity in widespread parieto-
\end{abstract}

\section{INTRODUCTION}

Neuroimaging researchers tacitly assume that body position has a negligible impact on human brain activity. However, postural discrepancies may hold important implications for brain function in general and for specific imaging methodologies in particular (Thibault \& Raz, 2016; Raz et al., 2005). Behavioral findings indicate that body posture alters perceptual thresholds and cognitive processing (Lipnicki \& Byrne, 2008; Lundström, Boyle, \& Jones-Gotman, 2008). Moreover, converging evidence demonstrates that posture regulates physiological factors, including hemodynamics, and influences concomitant neurocognitive function (Spironelli, Busenello, \& Angrilli, 2016; Thibault, Lifshitz, \& Raz, 2016; Thibault, Lifshitz, Jones, \& Raz, 2014; Benvenuti, Bianchin, \& Angrilli, 2013; Fardo, Spironelli, \& Angrilli, 2013; Rice, Rorden, Little, \& Parra, 2013; Chang et al., 2011; Spironelli \& Angrilli, 2011; Ouchi, Okada, Yoshikawa, Nobezawa, \& Futatsubashi, 1999; Cole, 1989). Comparing postures using a stanceadjustable PET gantry, one study reported signal differences across a range of cortical and subcortical regions

\footnotetext{
${ }^{1}$ McGill University, Montreal, Canada, ${ }^{2}$ Chapman University, Irvine, CA, ${ }^{3}$ Sir Mortimer B. Davis Jewish General Hospital, Montreal, Canada

*These authors contributed equally to the study.
}

occipital cortex. Moreover, sitting upright and reclining postures correlated with dampened activity in prefrontal regions across a range of bandwidths (i.e., from alpha to low gamma). The observed effects were large, with a mean Cohen's $d$ of 0.95 (SD = 0.23). In addition to neural activity, physiological parameters such as muscle tension and eye blinks may have contributed to these posture-dependent changes in brain signal. Regardless of the underlying mechanisms, however, the present results have important implications for the acquisition and interpretation of multimodal imaging data (e.g., studies combining fMRI or PET with EEG or MEG). More broadly, our findings indicate that generalizing results-from supine neuroimaging measurements to erect positions typical of ecological human behavior-would call for considering the influence that posture wields on brain dynamics.
(Ouchi et al., 1999). In addition, a few studies have found changes in EEG signals as a function of posture (Spironelli et al., 2016; Benvenuti et al., 2013; Fardo et al., 2013; Rice et al., 2013; Chang et al., 2011; Spironelli \& Angrilli, 2011; Cole, 1989). An EEG effort from our group indicated that orthostatic condition rapidly influences high-frequency electrical activity across the cortex (Thibault et al., 2014). In addition, we recently published a proof-of-concept analysis based on the present multipostural magnetoencephalography (MEG) data set (Thibault et al., 2016). However, this preliminary sensor level analysis could hardly elucidate how body position influences neural activity in specific anatomical areas. Thus, here we used a source localization approach to further examine the effects of posture at the level of regional brain function.

Body posture may impact neural function through a variety of physiological mechanisms. Gravity in the supine position stimulates baroreceptors that reduce sympathetic system activation (Mohrman \& Heller, 2003), decreasing noradrenergic output from the locus coeruleus (Berridge \& Waterhouse, 2003) and consequently dampening cortical excitability (Rau \& Elbert, 2001). In addition, supine posture modulates respiration, regardless of age, by altering diaphragm function (Rehder, 1998). This caveat holds special importance for independent component analysisbased measures of resting-state functional connectivity, which show substantial respiratory confounds (Birn, Smith, 
Jones, \& Bandettini, 2008). Such postural nuances come to the fore as researchers increasingly compare supine fMRI findings with resting-state electrophysiological data from EEG and intracranial recordings, often acquired in the upright position (Lei, Hu, \& Yao, 2012; Agam et al., 2011; Lei et al., 2011).

MEG is advantageous for studying the effects of posture on brain activity because certain MEG systems offer scanning capabilities across a range of body positions (see Figure 1). In contrast, although upright MRI scanners for humans exist, they tend to employ low magnetic fields that often preclude functional sequences. Moreover, whereas previous studies of posture used either PET or EEG, these methodologies lack integration of high-resolution spatial and temporal signals. On the one hand, PET provides reasonable spatial resolution but crude temporal resolution via an indirect measure of neural activity. On the other hand, EEG directly measures brain oscillations with millisecond precision but offers poorer signal localization due to smearing of electrical signals when passing through the cranial fluids and tissues (Vorwerk et al., 2014). Here we leveraged MEG localization analysis, which offers a direct measure of oscillatory activity with high spatiotemporal accuracy, to unravel the influence of body position on regional activity throughout the cortex.

\section{METHODS}

\section{Participants}

Twelve participants (mean age $=26.4 \pm 4.2$ years; six women) provided written informed consent in accordance with the research ethics board at the Montreal Neurological Institute and in compliance with the Declaration of Helsinki. Participants were right-handed, reported normal or corrected-to-normal vision, and received customary monetary compensation for their involvement.

\section{Procedure}

All sessions began with a 2-min empty room MEG recording. We then tested participants for magnetic artifacts in a brief preliminary MEG scan. For the main portion of the experiment, participants transitioned among three postures (sitting upright, reclining at $45^{\circ}$, and lying supine) in a counterbalanced fashion. For each posture, participants underwent two 8-min resting-state MEG scans, separated by a brief (1-2 min) break in the scanner. Throughout the MEG acquisitions, we instructed participants to relax, remain still, and fixate on a point directly ahead while keeping their eyes open. We standardized the visual environment by draping a white sheet around their visual field.

\section{Magnetoencephalography}

We acquired MEG data using the VSM/CTF system (MEG International Services Ltd., Coquitlam, Canada) at the Montreal Neurological Institute. The sensor array consisted of 270 axial gradiometers plus an additional nine reference magnetometers and 17 reference gradiometers farther from the helmet to remove environmental noise. We recorded using a sampling rate of $2400 \mathrm{~Hz}$ inside a dedicated scanning room with full three-layer passive magnetic shielding, while head-positioning coils and a 3-D digitizer system (Polhemus Isotrack, Colchester, VT) registered cephalic position throughout. In line with standard guidelines, we recorded electrocardiograms and electrooculograms to capture heartbeat and eye-blink artifacts (Gross et al., 2013). Between postures, participants left the scanning room while an experimenter adjusted the angle of the MEG dewar. We then waited for $15 \mathrm{~min}$, followed by a 2 -min empty room recording, to ensure that the liquid helium level outside the helmet and the temperature at the sensors had stabilized. On the basis of tests of our MEG system at the Montreal Neurological Institute, we determined that noise contamination from the sensors levels off within $15 \mathrm{~min}$ (Figure 2 displays empty room and participant-scan noise spectra for all dewar positions and body postures). Whereas the helium boil-off rate increases when the dewar is in the supine recording position, all sensors remain submerged in liquid helium and the temperature at each sensor remains constant.

Before the scans, we placed foam blocks between the helmet and the forehead of participants to help reduce

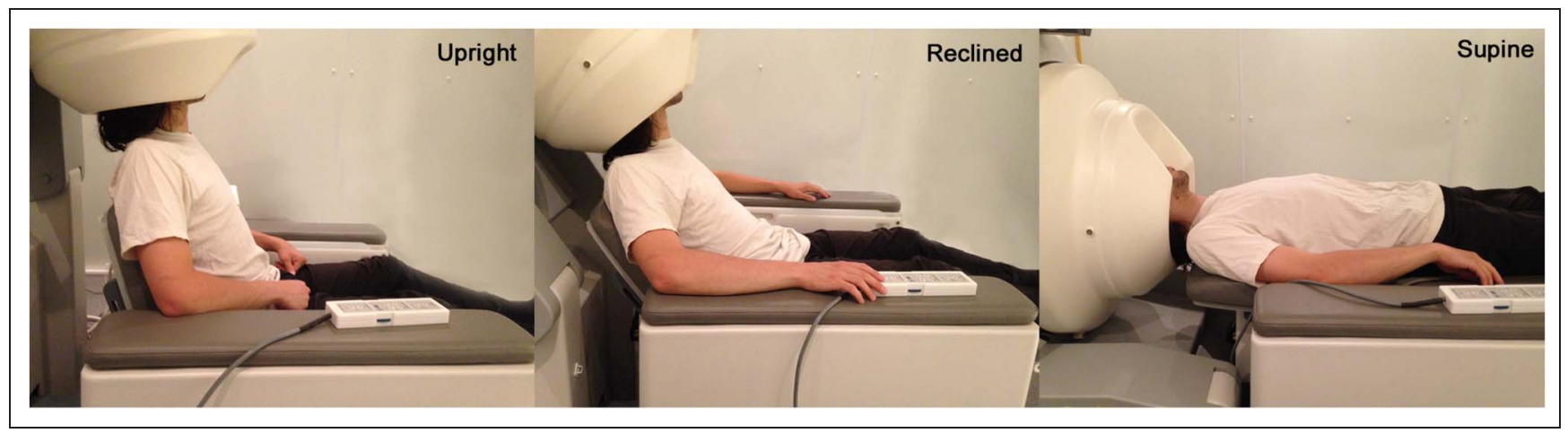

Figure 1. Posture and dewar positions. 


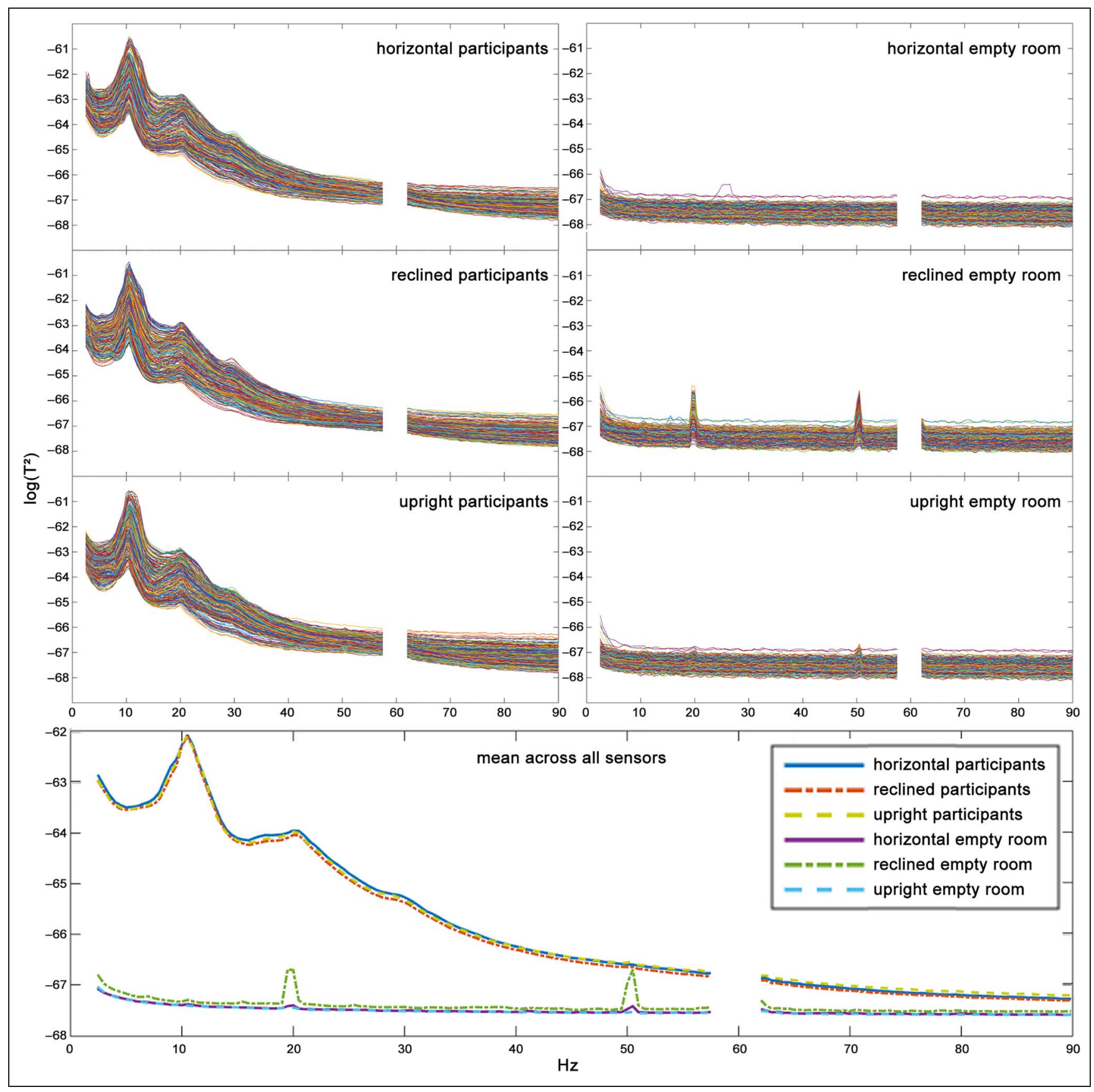

Figure 2. Noise spectra for participant and empty room recordings. The top six graphs depict the PSDs for each of the 270 gradiometers averaged across all runs for each posture (for participant recordings, on the left) and each dewar position (for empty room recordings, on the right). The bottom graph depicts the average across all 270 gradiometers for each of the above six conditions. As in our analysis, in this graph we removed frequencies below $2 \mathrm{~Hz}$ as well as electrical contamination from 58 to $62 \mathrm{~Hz}$. When performing our source analysis, we removed the environmental noise detected before each participant recording by accounting for an empty room noise covariance matrix. For example, this analysis regressed out the two blips around 20 and $50 \mathrm{~Hz}$ in the reclined empty room condition.

head motion, if needed. The exact placement of these foam blocks depended on the size and shape of the individual head. We instructed participants to position their head such that they were touching but not pressing against the top of the helmet. Recently acquired T1weighted anatomical MRI volumes helped map head position relative to the helmet. To facilitate the analysis, we down-sampled the high-resolution triangulated corti- cal surfaces to 15,000 vertices in line with standard protocol (Baillet, Mosher, \& Leahy, 2001).

\section{Data Processing}

We processed and analyzed MEG data using Brainstorm (Tadel, Baillet, Mosher, Pantazis, \& Leahy, 2011). We applied a high-pass filter at $0.1 \mathrm{~Hz}$ and removed potential 
electrical contamination using a sinusoidal (notch) filter at 60, 120, 180, and $240 \mathrm{~Hz}$. We then removed cardiac sources and contamination from eye blinks and eye movements by designing signal-space projectors (SSPs). Each SSP was specific to a particular run. We removed one cardiac and one blink SSP from each run to maintain comparable cleaning procedures and levels of background data subtracted between postures. We then visually inspected all data and discarded segments with any lingering ocular or cardiac contamination as well as high-amplitude muscle artifacts. We discarded data segments in which either of the two head localizer coils (left and right pre-auricular points) was farther than $5 \mathrm{~mm}$ from its position at the beginning of the recording; on average, participants moved their head less than $2 \mathrm{~mm}$ by the end of the recording (see Figure 3). We calculated a noise covariance matrix from each of the 36 empty room recordings (i.e., 12 participants by three postures). Each baseline noise recording was then applied to the corresponding participant recording to tease apart fluctuations in instrumental and environmental dynamics that the sensors detected in the empty room (Tadel et al., 2011). This procedure minimizes the potential influence of noise differences associated with different dewar positions.

We computed a head model of the cortex surface for each run using overlapping spheres and proceeded to compute sources using the whitened and depth-weighted linear L2-minimum norm estimates (wMNE) algorithm implemented in Brainstorm. To normalize sources across participants, we projected (warped) the sources from each participant onto the MNI/Colin27 template brain (Collins et al., 1998). The algorithms responsible for this transformation from sensor level data to source-space activity take into account head placement in relation to sensor location and thus compensate for differences in head size between participants and head placement across runs. We then calculated the power spectrum density (PSD) for each run at all 15,000 vertices on the template brain for delta $(\delta) 2-4 \mathrm{~Hz}$, theta $(\theta) 4-8 \mathrm{~Hz}$, alpha $(\alpha)$ 8-14 Hz, beta $(\beta)$ 14-30 Hz, low gamma $(\gamma 1)$ 30-58, and high gamma $(\gamma 2)$ 62-90 Hz using 50\% overlapping windows of 2-sec epochs. We then divided these 15,000 vertices into 68 cortical regions as per the Desikan-Killiany neuroanatomical atlas (Desikan et al., 2006). We averaged the PSDs across all the vertices in each scout to obtain 68 averaged PSDs.

\section{Statistical Analysis}

We first calculated the average of the two runs for each participant in each posture. Using the $\mathrm{R}$ statistics package, we conducted two-tailed paired sample $t$ tests on the logarithm of the power of the current density for
Figure 3. Head displacement in upright, reclined, and supine postures. This figure displays, as a function of posture, the means and standard deviations in head displacement of the left and right auricular head localizer units at every second of the 480-sec (8-min) recordings. On average, participants displaced their head by about twice as much when sitting upright (left: $1.7 \mathrm{~mm}$, right: $2.0 \mathrm{~mm}$ ) compared with when lying supine (left: $0.7 \mathrm{~mm}$, right: $1.1 \mathrm{~mm})$. However, mean head displacements in all postures remained well below the threshold $(\sim 5 \mathrm{~mm})$ that would call for repositioning the head or initiating a new head position file in standard MEG analysis practice (Gross et al., 2013; Whalen, Maclin, Fabiani, \& Gratton, 2008). Longer recordings and particular populations (e.g., children: Wehner et al., 2008) increase the likelihood of greater head displacement. In such experiments, posture may prove especially pertinent with respect to head movement.

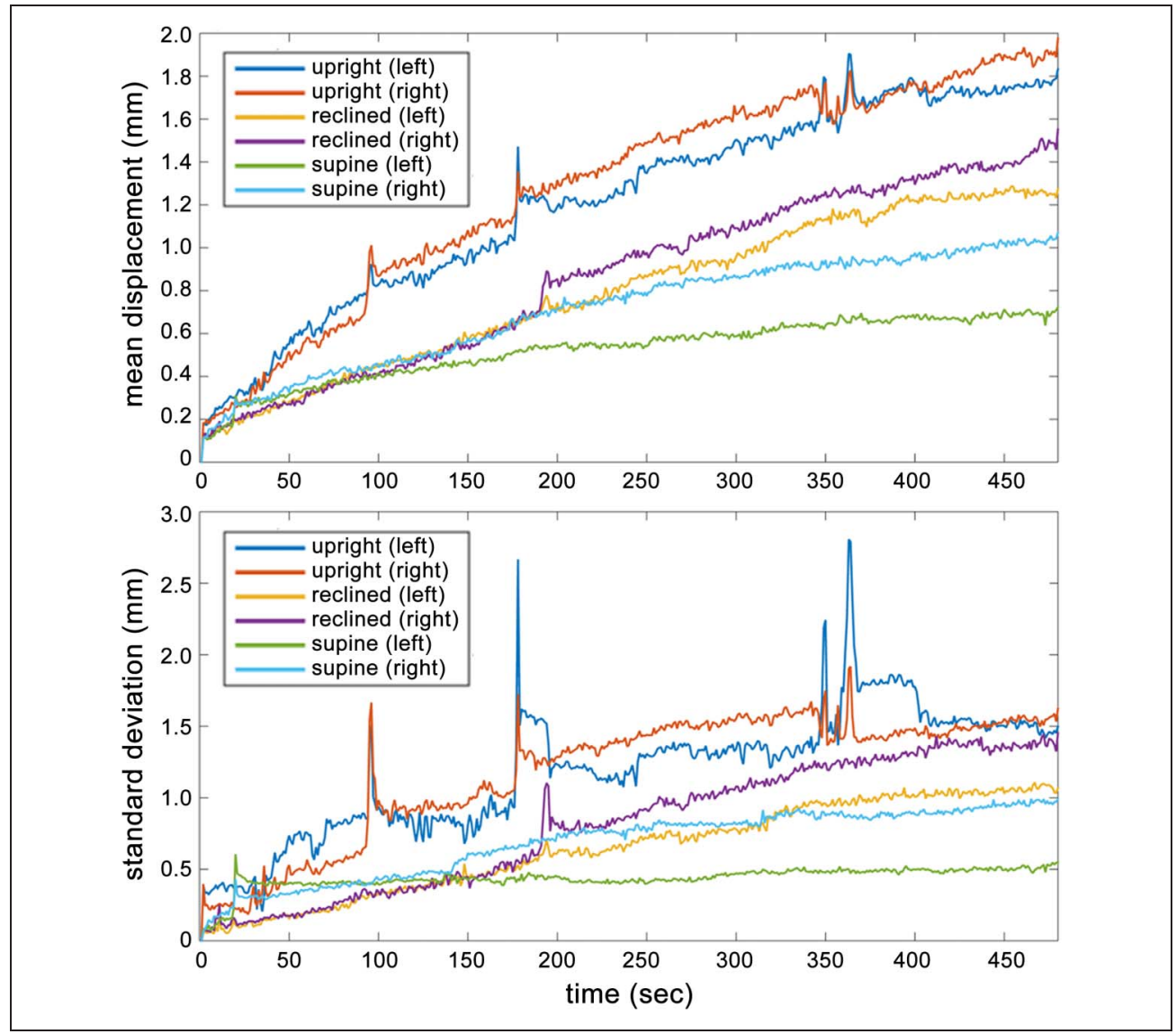




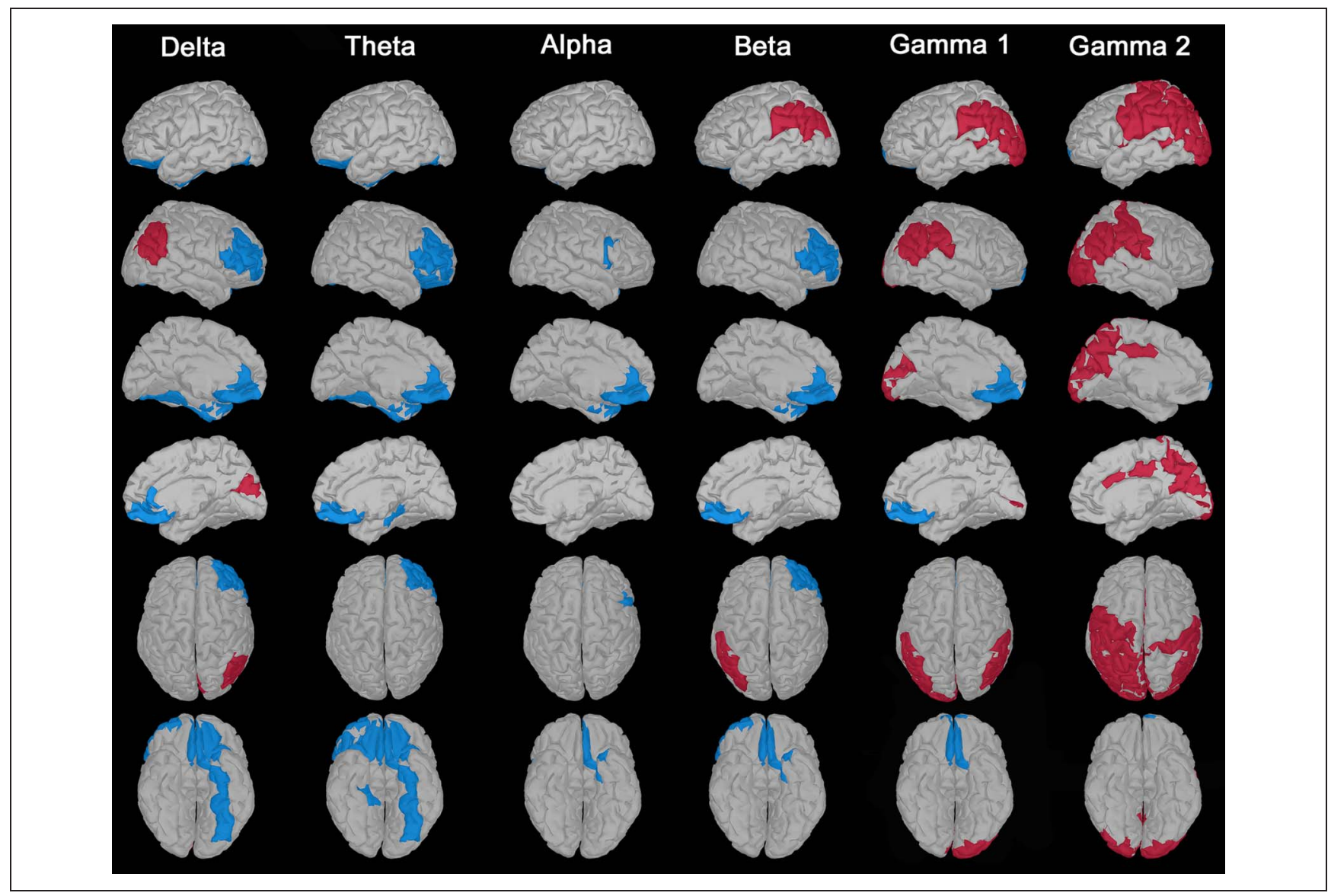

Figure 4. Posture-dependent changes in regional brain activity. Colored brain regions show areas where $t$ tests revealed source level power differences when contrasting sitting upright against lying supine (mapped on the Desikan-Killiany neuroanatomical atlas). Red $(q<0.05)$ signifies greater oscillatory activity when sitting upright, whereas blue $(q<0.05)$ signifies lower activity when sitting upright. Each column presents one brain map viewed from six different angles.

each Desikan-Killiany region (Desikan et al., 2006) for each bandwidth, for a total of 408 (68 regions by six bandwidths) $p$ values per contrast (i.e., sitting upright vs. lying supine, sitting upright vs. reclining at $45^{\circ}$, reclining at $45^{\circ}$ vs. lying supine). To account for multiple comparisons, we calculated adjusted $p$ values ( $q$ values) using the false discovery rate function from the qvalue package available in R (Storey, Bass, Dabney, \& Robinson, 2015) for each of the three contrasts. For the empty room recordings, we repeated this analysis for the PSD values obtained from each magnetic sensor, rather than from the Desikan-Killiany regions used for the participant recordings (due to the absence of a head in the dewar) and found no statistical difference between postures. We also performed one-tailed paired sample $t$ tests on heart rate for each contrast.

\section{RESULTS}

Our main contrast of interest investigated differences in oscillatory power across the whole brain between sitting upright and lying supine. For this contrast, we conducted $408 t$ tests ( 68 scout regions by six bandwidths). Of these tests, 76 yielded significant differences in brain signal (corrected for multiple comparisons, $q<0.05$ ). Figure 4 displays the significant brain regions and frequency bands for the contrast between sitting upright and lying supine. Table 1 further lists the anatomical label, effect size, and amplitude difference for each of the significant regions at each bandwidth. The mean Cohen's $d$ for these significant effects was $0.87(S D=0.28)$, indicating large effect sizes.

In addition to our primary analysis, we also investigated power differences associated with reclining by conducting two contrasts ( $408 t$ tests for each): (1) reclining versus lying supine and (2) reclining versus sitting upright. In the reclined versus supine contrast, 16 of the $408 t$ tests yielded significant changes in brain signal (corrected for multiple comparisons, $q<0.05$; see Table 2). The effects were large, with a mean Cohen's $d$ of $1.33(S D=0.28)$. Although this mean effect size was notably larger than in the sitting upright versus lying supine contrast, the amplitude of the changes was similar (compare Tables 1 and 2). Thus, the difference in effect size likely reflects a difference in variance. When comparing between sitting upright and reclining, we found a significant difference in 
Table 1. Sitting Upright versus Lying Supine

\begin{tabular}{|c|c|c|c|c|c|c|c|c|c|c|c|c|}
\hline \multirow[b]{2}{*}{ Desikan-Killiany Region } & \multicolumn{2}{|c|}{ Delta } & \multicolumn{2}{|c|}{ Theta } & \multicolumn{2}{|c|}{ Alpha } & \multicolumn{2}{|c|}{ Beta } & \multicolumn{2}{|c|}{ Gamma 1} & \multicolumn{2}{|c|}{ Gamma 2} \\
\hline & $d$ & $\Delta$ & $d$ & $\Delta$ & $d$ & $\Delta$ & $d$ & $\Delta$ & $d$ & $\Delta$ & $d$ & $\Delta$ \\
\hline L temporal pole & 0.92 & -0.50 & 1.08 & -0.41 & 0.74 & -0.32 & 0.75 & -0.32 & & & & \\
\hline L rostral anterior cingulate & 0.91 & -0.49 & 0.88 & -0.34 & 0.80 & -0.33 & 0.77 & -0.31 & 0.73 & -0.09 & & \\
\hline L medial orbitofrontal & 0.90 & -0.54 & 1.17 & -0.43 & 0.98 & -0.39 & 0.97 & -0.39 & 0.76 & -0.12 & & \\
\hline L fusiform & 0.88 & -0.34 & 0.79 & -0.26 & & & & & & & & \\
\hline $\mathrm{R}$ pars triangularis & 0.86 & -0.33 & 1.24 & -0.31 & & & 1.16 & -0.21 & & & & \\
\hline $\mathrm{R}$ inferior parietal & 0.86 & 0.29 & & & & & & & 0.82 & 0.16 & 0.99 & 0.14 \\
\hline L entorhinal & 0.85 & -0.44 & 0.79 & -0.35 & 0.90 & -0.28 & 0.85 & -0.28 & & & & \\
\hline $\mathrm{R}$ medial orbitofrontal & 0.80 & -0.41 & 0.94 & -0.31 & & & 0.83 & -0.27 & 0.73 & -0.11 & & \\
\hline L lateral orbitofrontal & 0.79 & -0.47 & 0.95 & -0.30 & & & & & & & & \\
\hline R cuneus & 0.78 & 0.42 & & & & & & & & & 0.74 & 0.18 \\
\hline $\mathrm{R}$ rostral anterior cingulate & 0.76 & -0.37 & & & & & & & & & & \\
\hline $\mathrm{R}$ rostral middle frontal & 0.76 & -0.36 & 0.91 & -0.31 & & & 0.76 & -0.29 & & & & \\
\hline R parahippocampal & & & 0.82 & -0.20 & & & & & & & & \\
\hline $\mathrm{R}$ pars orbitalis & & & 0.81 & -0.24 & & & & & & & & \\
\hline R lateral orbitofrontal & & & 0.76 & -0.25 & & & & & & & & \\
\hline $\mathrm{R}$ pars opercularis & & & & & 0.78 & -0.24 & & & & & & \\
\hline L inferior parietal & & & & & & & 0.80 & 0.31 & 0.85 & 0.24 & 1.22 & 0.20 \\
\hline L supramarginal & & & & & & & 0.72 & 0.20 & 0.90 & 0.25 & 1.04 & 0.28 \\
\hline $\mathrm{R}$ frontal pole & & & & & & & & & 1.33 & -0.24 & & \\
\hline L frontal pole & & & & & & & & & 1.11 & -0.26 & 0.71 & -0.18 \\
\hline R supramarginal & & & & & & & & & 1.06 & 0.12 & 1.22 & 0.15 \\
\hline $\mathrm{L}$ transverse temporal & & & & & & & & & 0.88 & 0.24 & 0.92 & 0.29 \\
\hline L banks sts & & & & & & & & & 0.80 & 0.22 & 0.99 & 0.24 \\
\hline L lateral occipital & & & & & & & & & 0.78 & 0.28 & 0.94 & 0.27 \\
\hline L pericalcarine & & & & & & & & & 0.76 & 0.28 & 0.85 & 0.27 \\
\hline $\mathrm{R}$ pericalcarine & & & & & & & & & 0.74 & 0.26 & 0.80 & 0.24 \\
\hline L cuneus & & & & & & & & & 0.72 & 0.26 & 0.80 & 0.20 \\
\hline L postcentral & & & & & & & & & & & 0.94 & 0.21 \\
\hline L posterior cingulate & & & & & & & & & & & 0.92 & 0.12 \\
\hline $\mathrm{R}$ posterior cingulate & & & & & & & & & & & 0.79 & 0.09 \\
\hline L precentral & & & & & & & & & & & 0.77 & 0.19 \\
\hline R caudal anterior cingulate & & & & & & & & & & & 0.77 & 0.09 \\
\hline $\mathrm{R}$ precuneus & & & & & & & & & & & 0.76 & 0.17 \\
\hline R postcentral & & & & & & & & & & & 0.75 & 0.09 \\
\hline L superior parietal & & & & & & & & & & & 0.74 & 0.14 \\
\hline $\mathrm{R}$ banks sts & & & & & & & & & & & 0.74 & 0.17 \\
\hline R lateral occipital & & & & & & & & & & & 0.71 & 0.23 \\
\hline L precuneus & & & & & & & & & & & 0.71 & 0.16 \\
\hline
\end{tabular}

This table lists Desikan-Killiany neuroanatomical regions that showed a significant difference in oscillatory power in the contrast of sitting upright versus lying supine $(q<0.05)$. Each result includes effect size (Cohen's $d$ ) and the amplitude of the difference $(\Delta)$ in $\log \left((A-m)^{2}\right)$. 
Table 2. Reclining versus Lying Supine

\begin{tabular}{|c|c|c|c|c|c|c|c|c|c|c|c|c|}
\hline \multirow[b]{2}{*}{ Desikan-Killiany Region } & \multicolumn{2}{|c|}{ Delta } & \multicolumn{2}{|c|}{ Theta } & \multicolumn{2}{|c|}{ Alpha } & \multicolumn{2}{|c|}{ Beta } & \multicolumn{2}{|c|}{ Gamma 1} & \multicolumn{2}{|c|}{ Gamma 2} \\
\hline & $d$ & $\Delta$ & $d$ & $\Delta$ & $d$ & $\Delta$ & $d$ & $\Delta$ & $d$ & $\Delta$ & $d$ & $\Delta$ \\
\hline L temporal pole & 1.15 & -0.48 & & & & & & & & & & \\
\hline $\mathrm{R}$ medial orbitofrontal & 1.20 & -0.44 & & & & & & & & & & \\
\hline $\mathrm{R}$ rostral anterior cingulate & 1.20 & -0.47 & & & & & & & & & & \\
\hline L lateral orbitofrontal & 1.25 & -0.48 & 1.14 & -0.26 & & & & & & & & \\
\hline L medial orbitofrontal & 1.28 & -0.65 & 1.67 & -0.44 & 1.58 & -0.37 & & & & & & \\
\hline $\mathrm{L}$ insula & 1.26 & -0.27 & & & & & & & & & & \\
\hline L lingual & 1.27 & -0.25 & & & & & & & & & & \\
\hline L rostral anterior cingulate & 1.41 & -0.53 & & & & & & & & & & \\
\hline $\mathrm{L}$ inferior temporal & 1.48 & -0.36 & & & & & & & & & & \\
\hline L fusiform & 1.85 & -0.35 & 1.27 & -0.26 & & & & & & & & \\
\hline L parahippocampal & & & 1.13 & -0.22 & & & & & & & & \\
\hline $\mathrm{R}$ frontal pole & & & & & & & & & 1.10 & -0.23 & & \\
\hline
\end{tabular}

This table lists Desikan-Killiany neuroanatomical regions that showed a significant difference in oscillatory power in the contrast of reclining versus lying supine $(q<0.05)$. Each result includes effect size (Cohen's $d)$ and the amplitude of the difference $(\Delta) \log \left((A-m)^{2}\right)$.

only one region at one bandwidth. For a comprehensive list of all effects, including nonsignificant effects, please see the Supplementary Materials (available from https:// razlab.org/links/koSxUa).

Heart rate was greater in more upright postures (sitting upright: 70.7 beats per minute; reclining: $68.8 \mathrm{bpm}$; lying supine: $64.6 \mathrm{bpm}$ ). Using a Bonferroni corrected $\alpha=.017$, only the difference between sitting upright and lying supine $(p<.001, d=1.33)$ and reclining and lying supine $(p=.015, d=0.72)$ met significance (sitting upright vs. reclining: $p=.05, d=0.52$ ). As an exploratory post hoc analysis, we tested whether between-posture differences in heart rate correlated with between-posture differences in oscillatory power (using Pearson's correlation coefficients at each neuroanatomical region and bandwidth). These analyses yielded no significant correlations, yet the results remain inconclusive because the relationship between heart rate and brain activity may be nonlinear and this analysis underpowered.

\section{DISCUSSION}

This study leverages multipostural MEG to unravel how body orientations associated with prevalent imaging procedures (e.g., sitting upright in EEG vs. lying supine in fMRI) impact resting-state brain activity. Extending previous sensor level findings (Spironelli et al., 2016; Thibault et al., 2014, 2016; Benvenuti et al., 2013; Fardo et al., 2013; Rice et al., 2013; Chang et al., 2011; Spironelli \& Angrilli, 2011; Cole, 1989), here we report a source level MEG analysis revealing that sitting upright, compared with lying supine, was associated with greater power in high-frequency bands (i.e., extending from beta to high gamma) in a wide swath of parieto-occipital cortex. Furthermore, prefrontal oscillatory power was dampened in the upright-seated position to varying degrees depending on the bandwidth (with effects ranging from delta to low gamma bands). Beyond our primary analysis contrasting sitting upright versus lying supine, we also investigated brain activity associated with reclining at $45^{\circ}$. When comparing reclined to supine posture, we found power differences in frontal regions, which largely overlapped with the effects from the lying supine versus sitting upright contrast. On the other hand, we hardly found any significant brain changes between reclining and sitting upright. Effect sizes were large across all significant tests, with a mean Cohen's $d$ of $0.95(S D=0.23)$. This overarching pattern of results indicates that the oscillatory dynamics of the resting brain differ dramatically between supine posture and more upright body positions.

EEG studies have associated high-frequency activity with cognitive processing (e.g., alert mental states; Kaiser \& Lutzenberger, 2005) and lower-frequency activity with relaxation (e.g., drowsy states; Strijkstra, Beersma, Drayer, Halbesma, \& Daan, 2003). Thus, the present findings suggest that the brain may linger in a mode of decreased vigilance when supine compared with when upright. In line with this interpretation and related accounts (e.g., Jones \& Dean, 2004), our electrocardiogram data showed lower heart rate in the supine posture.

Our present findings accord with previous reports investigating the influence of posture on resting-state brain function. The collective evidence indicates that upright postures are associated with greater power in highfrequency bands (Thibault et al., 2014; Chang et al., 2011; 
Cole 1989) and reduced power in low-frequency bands (Spironelli et al., 2016; Chang et al., 2011). Our earlier sensor level analysis of the current data set also showed high-gamma increases in more upright postures, but these effects were restricted to smaller regions of the left hemisphere (Thibault et al., 2016). In sensor level MEG analysis, however, variation in head distance from the sensors presents a substantial confound (see discussion in Thibault et al., 2016). This methodological caveat may explain why our earlier sensor level analysis was unable to pick up the more robust, distributed effects we observed here using a source localization approach.

In terms of regional brain activity, our present observations coalesce with the findings of an earlier account using multipostural PET (Ouchi et al., 1999). Congruent with our observation of greater upright gamma power in parieto-occipital areas, this PET study reported increased cerebral blood flow to visual areas when standing upright compared with when lying down. Yet, here we observed a more distributed pattern of high-frequency activityextending beyond the visual areas to a large portion of posterior cortex. In addition, we found differences in lowfrequency bands among frontal regions, which were absent from our previous EEG results (Thibault et al., 2014). In that EEG study, however, participants assumed body positions for shorter time periods and received instructions from experimenters every $30 \mathrm{sec}$ to change behavioral condition (e.g., to open or close their eyes for the next block). Thus, participants may have had less time to settle into a relaxed state during the supine position, which may have limited the differences in low-frequency power between postures. Of particular interest, the present source analysis revealed alterations in core regions of the default-mode network (e.g., posterior cingulate, precuneus, inferior parietal lobule, parahippocampus, rostral anterior cingulate), which has been proposed as a central hub of anatomical and functional organization in the human brain (van den Heuvel \& Sporns, 2013).

Certain physiological parameters may have played a role in shaping our results. Muscle activity exerts an influence on signals in the gamma range (Muthukumaraswamy, 2013) and thus might have contributed to the posterior high-frequency activity. In addition, persistent eye-blink artifacts might have survived our standard data cleaning procedures and thus contributed to the orbital activity. On the other hand, systematic differences in head position are unlikely to explain the present results. Although the back of the head might have been closer to the sensors in the supine position, our source-space analysis accounts for variations in head placement using participant-specific 3-D digitization of cranium size and shape, head localizer coils, and MRI-guided anatomy per individual. Moreover, we would expect opposing results in frontal versus parietooccipital regions if a consistent shift toward occipital head placement drove neural difference. In particular, we would expect higher occipital signal in the supine posture because these regions would be closer to the sensors. In- stead, our results show lower parieto-occipital power in the supine posture. Regardless of the underlying causes, the alterations we observed hold broad implications for the field of neuroimaging.

Orthostatic caveats take on particular importance as the domain of cognitive neuroscience moves toward triangulating data from multiple imaging modalities involving different body stances (Calhoun \& Sui, 2016; Garcés et al., 2016; Agam et al., 2011; Lei et al., 2011). Even within the realm of MEG, posture varies from study to study: whereas upright positions are most typical, supine measurements are also common in multimodal imaging contexts (e.g., Carhart-Harris et al., 2016; Larson-Prior et al., 2013) and when investigating specific clinical populations (e.g., epilepsy: Pellegrino et al., 2016; multiple sclerosis: Schoonheim et al., 2013). Unfortunately, many a MEG report neglects to specify the acquisition posture. In addition, our main finding that upright posture is associated with higher parieto-occipital gamma power has direct relevance for comparisons between upright EEG/ MEG data and supine fMRI scans. Intracranial, EEG, and MEG recordings have implicated gamma band activity in a host of cognitive functions including attention, memory, and sensory processing (Jensen, Kaiser, \& Lachaux, 2007). Moreover, our MEG data are relevant for fMRI studies because the BOLD signal, which serves as a proxy for neural activity in fMRI, correlates with gamma activity (Nir et al., 2007; Niessing et al., 2005). Our findings thus highlight how sitting upright or lying down-body positions associated with common imaging modalitiesimpact the resultant data acquired via those technologies. The current account paves the road to a more scientific understanding of posture as a ubiquitous, albeit little acknowledged, procedural caveat in cognitive neuroscience research.

\section{Acknowledgments}

Michael Lifshitz acknowledges a Francisco J. Varela Research Award from the Mind and Life Institute and a Vanier Canada Graduate Scholarship from the Natural Sciences and Engineering Research Council of Canada (NSERC). Robert Thibault acknowledges an Alexander Graham Bell Canada Graduate Scholarship from NSERC and funding from the Bial Foundation. Amir Raz acknowledges funding from the Canada Research Chair program, Discovery and Discovery Acceleration Supplement grants from NSERC, the Canadian Institutes of Health Research, and the Bial Foundation. We thank Devin Terhune, Sylvain Baillet, Elizabeth Bock, and Ishan Walpola for assistance with experimental design and data collection.

Reprint requests should be sent to Professor Amir Raz, Institute for Community and Family Psychiatry, 4333 Cote-Ste-Catherine Rd., Montreal, QC, H3T1E4, Canada, or via e-mail: amir.raz@ mcgill.ca.

\section{REFERENCES}

Agam, Y., Hämäläinen, M. S., Lee, A. K. C., Dyckman, K., Friedman, J. S., Isoma, M., et al. (2011). Multimodal neuroimaging 
dissociates hemodynamic and electrophysiological correlates of error processing. Proceedings of the National Academy of Sciences, U.S.A., 108, 17556-17561.

Baillet, S., Mosher, J. C., \& Leahy, R. M. (2001). Electromagnetic brain mapping. IEEE Signal Processing Magazine, 18, 14-30.

Benvenuti, S. M., Bianchin, M., \& Angrilli, A. (2013). Posture affects emotional responses: A head down bed rest and ERP study. Brain and Cognition, 82, 313-318.

Berridge, C. W., \& Waterhouse, B. D. (2003). The locus coeruleus-noradrenergic system: Modulation of behavioral state and state-dependent cognitive processes. Brain Research Brain Research Reviews, 42, 33-84.

Birn, R. M., Smith, M. A., Jones, T. B., \& Bandettini, P. A. (2008). The respiration response function: The temporal dynamics of fMRI signal fluctuations related to changes in respiration. Neuroimage, 40, 644-654.

Calhoun, V. D., \& Sui, J. (2016). Multimodal fusion of brain imaging data: A key to finding the missing link(s) in complex mental illness. Biological Psychiatry: Cognitive Neuroscience and Neuroimaging, 1, 230-244.

Carhart-Harris, R. L., Muthukumaraswamy, S., Roseman, L., Kaelen, M., Droog, W., Murphy, K., et al. (2016). Neural correlates of the LSD experience revealed by multimodal neuroimaging. Proceedings of the National Academy of Sciences, U.S.A., 113, 4853-4858.

Chang, L.-J., Lin, J.-F., Lin, C.-F., Wu, K.-T., Wang, Y.-M., \& Kuo, C.-D. (2011). Effect of body position on bilateral EEG alterations and their relationship with autonomic nervous modulation in normal subjects. Neuroscience Letters, 490, 96-100.

Cole, R. J. (1989). Postural baroreflex stimuli may affect EEG arousal and sleep in humans. Journal of Applied Physiology, 67, 2369-2375.

Collins, D. L., Zijdenbos, A. P., Kollokian, V., Sled, J. G., Kabani, N. J., Holmes, C. J., et al. (1998). Design and construction of a realistic digital brain phantom. IEEE Transactions on Medical Imaging, 17, 463-468.

Desikan, R. S., Ségonne, F., Fischl, B., Quinn, B. T., Dickerson, B. C., Blacker, D., et al. (2006). An automated labeling system for subdividing the human cerebral cortex on MRI scans into gyral based regions of interest. Neuroimage, 31, 968-980.

Fardo, F., Spironelli, C., \& Angrilli, A. (2013). Horizontal body position reduces cortical pain-related processing: Evidence from late ERPs. PLoS One, 8, e81964.

Garcés, P., Pereda, E., Hernández-Tamames, J. A., Del-Pozo, F., Maestú, F., \& Ángel Pineda-Pardo, J. (2016). Multimodal description of whole brain connectivity: A comparison of resting state MEG, fMRI, and DWI. Human Brain Mapping, 37, 20-34.

Gross, J., Baillet, S., Barnes, G. R., Henson, R. N., Hillebrand, A., Jensen, O., et al. (2013). Good practice for conducting and reporting MEG research. Neuroimage, 65, 349-363.

Jensen, O., Kaiser, J., \& Lachaux, J.-P. (2007). Human gammafrequency oscillations associated with attention and memory. Trends in Neurosciences, 30, 317-324.

Jones, A. Y., \& Dean, E. (2004). Body position change and its effect on hemodynamic and metabolic status. Heart \& Lung: The Journal of Acute and Critical Care, 33, 281-290.

Kaiser, J., \& Lutzenberger, W. (2005). Human gamma-band activity: A window to cognitive processing. NeuroReport, 16, 207-211.

Larson-Prior, L. J., Oostenveld, R., Della Penna, S., Michalareas, G., Prior, F., Babajani-Feremi, A., et al. (2013). Adding dynamics to the Human Connectome Project with MEG. Neuroimage, 80, 190-201.

Lei, X., Hu, J., \& Yao, D. (2012). Incorporating fMRI functional networks in EEG source imaging: A Bayesian model comparison approach. Brain Topography, 25, 27-38.
Lei, X., Xu, P., Luo, C., Zhao, J., Zhou, D., \& Yao, D. (2011). fMRI functional networks for EEG source imaging. Human Brain Mapping, 32, 1141-1160.

Lipnicki, D. M., \& Byrne, D. G. (2008). An effect of posture on anticipatory anxiety. The International Journal of Neuroscience, 118, 227-237.

Lundström, J. N., Boyle, J. A., \& Jones-Gotman, M. (2008). Body position-dependent shift in odor percept present only for perithreshold odors. Chemical Senses, 33, 23-33.

Mohrman, D. E., \& Heller, L. J. (2003). Cardiovascular physiology. New York: Lange Medical Books/McGraw-Hill.

Muthukumaraswamy, S. D. (2013). High-frequency brain activity and muscle artifacts in MEG/EEG: A review and recommendations. Frontiers in Human Neuroscience, 7, 138.

Niessing, J., Ebisch, B., Schmidt, K. E., Niessing, M., Singer, W., \& Galuske, R. A., et al. (2005). Hemodynamic signals correlate tightly with synchronized gamma oscillations. Science, 309, 948-951.

Nir, Y., Fisch, L., Mukamel, R., Gelbard-Sagiv, H., Arieli, A., Fried, I., et al. (2007). Coupling between neuronal firing rate, gamma LFP, and BOLD fMRI is related to interneuronal correlations. Current Biology, 17, 1275-1285.

Ouchi, Y., Okada, H., Yoshikawa, E., Nobezawa, S., \& Futatsubashi, M. (1999). Brain activation during maintenance of standing postures in humans. Brain, 122, 329-338.

Pellegrino, G., Hedrich, T., Chowdhury, R., Hall, J. A., Lina, J. M., Dubeau, F., et al. (2016). Source localization of the seizure onset zone from ictal EEG/MEG data. Human Brain Mapping, 37, 2528-2546.

Rau, H., \& Elbert, T. (2001). Psychophysiology of arterial baroreceptors and the etiology of hypertension. Biological Psychology, 57, 179-201.

Raz, A., Lieber, B., Soliman, F., Buhle, J., Posner, J., Peterson, B. S., et al. (2005). Ecological nuances in functional magnetic resonance imaging (fMRI): Psychological stressors, posture, and hydrostatics. Neuroimage, 25, 1-7.

Rehder, K. (1998). Postural changes in respiratory function. Acta Anaesthesiologica Scandinavica, 42, 13-16.

Rice, J. K., Rorden, C., Little, J. S., \& Parra, L. C. (2013). Subject position affects EEG magnitudes. Neuroimage, 64, 476-484.

Schoonheim, M. M., Geurts, J. J., Landi, D., Douw, L., van der Meer, M. L., Vrenken, H., et al. (2013). Functional connectivity changes in multiple sclerosis patients: A graph analytical study of MEG resting state data. Human Brain Mapping, 34, 52-61.

Spironelli, C., \& Angrilli, A. (2011). Influence of body position on cortical pain-related somatosensory processing: An ERP study. PLoS One, 6, e24932.

Spironelli, C., Busenello, J., \& Angrilli, A. (2016). Supine posture inhibits cortical activity: Evidence from delta and alpha EEG bands. Neuropsychologia, 89, 125-131.

Storey, J. D., Bass, A. J., Dabney, A., \& Robinson, D. (2015). qualue: Q-value estimation for false discovery rate control. R Package Version 3.3.0 (http://github.com/jdstorey/qvalue).

Strijkstra, A. M., Beersma, D. G., Drayer, B., Halbesma, N., \& Daan, S. (2003). Subjective sleepiness correlates negatively with global alpha $(8-12 \mathrm{~Hz})$ and positively with central frontal theta $(4-8 \mathrm{~Hz})$ frequencies in the human resting awake electroencephalogram. Neuroscience Letters, 340, $17-20$.

Tadel, F., Baillet, S., Mosher, J. C., Pantazis, D., \& Leahy, R. M. (2011). Brainstorm: A user-friendly application for MEG/EEG analysis. Computational Intelligence and Neuroscience, 2011, 879716

Thibault, R., Lifshitz, M., \& Raz, A. (2016). Body position alters human resting state: Insights from multi-postural 
magnetoencephalography. Brain Imaging and Behavior, 10, 772-780.

Thibault, R. T., Lifshitz, M., Jones, J. M., \& Raz, A. (2014). Posture alters human resting-state. Cortex, 58, 199-205.

Thibault, R. T., \& Raz, A. (2016). Imaging posture veils neural signals. Frontiers in Human Neuroscience, 10, 520.

van den Heuvel, M. P., \& Sporns, O. (2013). Network hubs in the human brain. Trends in Cognitive Sciences, 17, 683-696.

Vorwerk, J., Cho, J.-H., Rampp, S., Hamer, H., Knösche, T. R., \& Wolters, C. H. (2014). A guideline for head volume conductor modeling in EEG and MEG. Neuroimage, 100, 590-607.

Whalen, C., Maclin, E. L., Fabiani, M., \& Gratton, G. (2008). Validation of a method for coregistering scalp recording locations with 3D structural MR images. Human Brain Mapping, 29, 1288-1301.

Wehner, D. T., Hämäläinen, M. S., Mody, M., \& Ahlfors, S. P. (2008). Head movements of children in MEG: Quantification, effects on source estimation, and compensation. Neuroimage, 40, 541-550. 